\title{
Posibilidades de reproducción de Schinus johnstonii (Anacardiaceae), una especie nativa del Monte argentino
}

\author{
Reproductive possibilities of Schinus johnstonii (Anacardiaceae), \\ a native species to the Argentine Monte
}

Diaz Arias, Daniela'; Yanina A. Torres ${ }^{1,2 *}$; Leticia S. Ithurrart1;

Daniela S. Cadillo 3

1 Departamento de Agronomía, Universidad Nacional del Sur (UNS), San Andrés 800, (8000) Bahía Blanca, Buenos Aires, Argentina.

2 Comisión de Investigaciones Científicas de la Provincia de Buenos Aires (CICPBA), Argentina.

3 Consejo Nacional de Investigaciones Científicas y Técnicas de Argentina (CONICET).

* Autor corresponsal: yatorres@criba.edu.ar

ORCID:

Daniela Diaz Arias: https://orcid.org/0000-0003-2095-869X

Yanina Alejandra Torres: https://orcid.org/0000-0002-4129-0695

Leticia Soledad Ithurrart: https://orcid.org/0000-0002-5651-9763

Daniela Solange Cardillo: https://orcid.org/0000-0002-9873-7945

\section{RESUMEN}

Schinus johnstonii F. A. Barkley (Anacardiaceae) es un arbusto nativo de la Provincia Fitogeográfica del Monte, donde representa un importante componente del pastizal natural. Su tolerancia a la sequía, junto a la presencia de una floración abundante, la convierten en una buena opción para proyectos paisajísticos y de restauración de ambientes degradados y deforestados. Para tales fines es necesario contar con información acerca de sus posibilidades de reproducción. Los objetivos de este trabajo fueron determinar los requerimientos básicos para la germinación de sus semillas (reproducción sexual), en condiciones de laboratorio, la viabilidad de las mismas y analizar la supervivencia de estacas (reproducción vegetativa), en condiciones controladas y bajo distintos tratamientos. Las semillas recolectadas se sometieron a seis tratamientos pre-germinativos: exocarpo intacto (control), remoción de exocarpo (desnudas), semillas desnudas con escarificación mecánica, semillas desnudas con escarificación química, semillas desnudas en remojo y semillas desnudas con exposición a humo. Sólo se observó germinación en semillas sin exocarpo. Las semillas desnudas germinaron a partir de los 10 días, con un valor máximo del 38\%, sin encontrarse diferencias entre los tratamientos. Se observó, además, una importante

Ref. bibliográfica: Diaz Arias, D.; Torres, Y. A.; Ithurrart, L. S.; Cadillo, D. S. 2020. "Posibilidades de reproducción de Schinus johnstonii (Anacardiaceae), una especie nativa del Monte argentino". Lilloa 57 (2): 125-143. Fundación Miguel Lillo, Tucumán, Argentina. D.O.I.: doi.org/10.30550/j.lil/2020.57.2/4 - Recibido: 4 de agosto 2020 - Aceptado: 6 de noviembre 2020

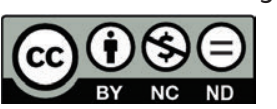


proporción de semillas no viables o vacías (30-40\%). El estudio de reproducción vegetativa, con estacas recolectadas durante los estadios fenológicos vegetativo y reproductivo, con y sin hormona de enraizamiento, dio como resultado un porcentaje bajo de rebrote (20\%), sin diferencias entre tratamientos. Según los resultados, las semillas de $S$. johnstonii presentarían dormición física impuesta por la cubierta (superada por remoción del exocarpo). La reproducción de este arbusto no sería recomendable por medio de estacas, aunque se sugiere realizar más estudios.

Palabras clave - Dormición; reproducción vegetativa; tratamientos pre-germinativos; viabilidad.

\begin{abstract}
Schinus johnstonii F. A. Barkley (Anacardiaceae) is a native shrub to the Monte Phytogeographic Province, where it represents an important component of rangelands. Its tolerance to drought and abundant flowering, render it a good option for landscaping and land restoration projects on degraded and deforested areas. For such purposes, information about its reproductive possibilities is needed. The objectives of this work were to determine the basic requirements for seed germination (sexual reproduction) under laboratory conditions, viability and to analyze the survival of cuttings (vegetative reproduction), under controlled conditions and different treatments. The collected seeds were subjected to six pre-germination treatments: intact exocarp (control), exocarp removal (bare), bare seeds with mechanical scarification, bare seeds with chemical scarification, bare seeds with soaking and bare seeds with exposure to smoke. Germination was only observed in seeds without exocarp. Bare seeds germinated after 10 days, with a maximum value of $38 \%$, with no differences between treatments. In addition, a significant proportion of non-viable or empty seeds (30-40\%) was observed. The vegetative reproductive study, with cuttings collected during the vegetative and reproductive phenological stages, with and without rooting hormone, resulted in a low percentage of regrowth (20\%), with no differences between treatments. According to these results, seeds of $S$. johnstonii would present physical dormancy imposed by the seed coat (overcome by the removal of the exocarp). The reproduction of this shrub by cuttings is not recommended, although further studies are suggested.
\end{abstract}

Keywords - Dormancy; vegetative reproduction; pre-germination treatments; viability.

\title{
INTRODUCCIÓN
}

Los ecosistemas áridos y semiáridos del mundo se encuentran bajo profundos procesos de degradación como consecuencia de diferentes factores naturales y antrópicos que interactúan y se retroalimentan entre sí. Argentina no es la excepción y particularmente la Provincia Fitogeográfica del Monte, es una región altamente susceptible de experimentar procesos erosivos, hídricos y eólicos, de diversa intensidad debido a 
la fragilidad que presenta (Villagra et al., 2009). En Buenos Aires, el Partido de Patagones posee el área de Monte nativo de mayor magnitud de la provincia (Giorgetti, Montenegro, Rodríguez, Busso, 2006). Sin embargo, este ecosistema se encuentra en riesgo a causa de la deforestación, el sobrepastoreo, las condiciones climáticas y las interacciones entre estos factores (Villagra et al., 2004). Además, el empleo del fuego como herramienta de manejo del pastizal, sin un planeamiento previo, favorece al empobrecimiento de la vegetación nativa, y con ello a la pérdida de diversidad. En ambientes tan frágiles la preservación del elemento leñoso, que sirve como esqueleto estructural de los componentes herbáceos, resulta imprescindible para su conservación (Bogino et al., 2002).

Dentro del componente leñoso del Monte se encuentra Schinus johnstonii F. A. Barkley (Anacardiaceae), una especie arbustiva endémica, sumamente resistente a la sequía y con potencial ornamental (Codina, Carrieri, Manzano, Fioretti, 2003; Steibel y Troiani, 2008). El molle es un arbusto perenne de entre 0,6 y 2 metros de altura. Sus ramas jóvenes, castaño-rojizas, terminan en espina; posee hojas simples, alternas o agrupadas y coriáceas. Las flores son pequeñas, amarillas, agrupadas en racimos. Su fruto es de tipo drupáceo oscuro, globoso, con pericarpio papiráceo y mesocarpo carnoso, de 7-8 mm de diámetro (Steibel y Troiani, 2008; Silva-Luz et al., 2018). Es una especie con propiedades medicinales (Doria, 2010), de madera dura y resistente, además de melífera (Pensiero, Muñoz, Martínez, 2005). Su valor como especie forrajera es reducido pero sus frutos son comestibles para aves y herbívoros domésticos y silvestres (Martín, Nicosia, Colombo, Lucas, 2001). La germinación se produce entre agosto y octubre, la floración en octubre-noviembre, la fructificación en noviembre y la dispersión de las semillas a fines de noviembre-diciembre (Giorgetti, Manuel, Montenegro, Rodríguez, Busso, 2000; Steibel y Troiani, 2008).

Las especies adaptadas a la heterogeneidad ambiental del Monte han desarrollado una amplia variedad de formas y adaptaciones morfológicas, anatómicas y fisiológicas que determinan distintos grados de xerofitismo (Villagra et al., 2011). Los mecanismos que les permiten a estas especies tolerar distintos tipos de estrés se manifiestan en diferentes etapas de la ontogenia, fundamentalmente en el período comprendido entre la germinación y el establecimiento. En el Monte, es entre estas dos etapas, cuando se producen los mayores porcentajes de mortalidad de plantas, tanto bajo condiciones naturales como en reforestaciones asistidas (Villagra et al., 2004). Estos mecanismos adaptativos, que ofrecen rusticidad a las especies nativas, también representan una limitante al momento de intentar la recuperación de áreas degradadas, debido al desconocimiento de la forma adecuada de propagación. Esta situación ha generado un creciente interés, tanto científico como comercial, en la búsqueda de formas de reproducción ex situ de las especies nativas, que permitan obtener ejemplares aptos para proyectos de restauración y de paisajismo local.

La latencia o dormición es el estado en el cual una semilla viable no germina, aunque se coloque en condiciones de humedad, temperatura y concentración de oxígeno adecuados (Young y Young, 1999). Ésta puede ser endógena, asociada a la condición del embrión mismo, o exógena, asociada a los efectos de la cubierta del fruto persistente que protege a la semilla. A su vez esta última se puede dividir en mecánica, química y física (Young y Young, 1999). Es habitual en algunas especies 
leñosas del Monte como Prosopis alpataco (Vega Riveros, Meglioli, Villagra, 2012), Geoffroea decorticans (Pece, Sobrero, Acosta, Rossi, 2014) y Condalia microphylla (Peláez, Boó, Elía, 1996), que las semillas presenten algún tipo de dormición física o mecánica debida a la dureza e impermeabilidad de sus cubiertas que impiden la germinación (Villagra et al., 2004). En la Familia Anacardiaceae, se ha encontrado la presencia de algún tipo de dormición en semillas de diversas especies. Para $R$ hus lanceolata (Rasmussen y Wright, 1988), R. glabra y R. aromática (Li, Baskin, Baskin, 1999a), Schinus molle (Corkidi, Cacho, Búrquez, 1991), Lithrea caustica (León-Lobos y Kalin-Arroyo, 1994) y Cotinus obovatus (Young y Young, 1999), se cita dormición física impuesta por la cubierta. La presencia de inhibidores químicos también puede condicionar la germinación de disemínulos en plantas superiores (Young y Young, 1999). En el género Schinus, la drupa posee un endocarpo compuesto de tres capas de esclereidas, que permanece adherido a las semillas (Wannan, 2006). Se ha propuesto que esta estructura sería la causante de la dormición física encontrada en algunas semillas (Li, Baskin, Baskin, 1999b), lo que haría presuponer la existencia de algún tipo de latencia impuesta por la cubierta también en S. johnstonii.

Existen diferentes métodos pre-germinativos ya probados que contribuyen a superar la dormición de las semillas. Estos incluyen la remoción o abrasión física de la cubierta seminal (sin dañar al embrión), la abrasión con algún medio ácido que debilite la testa (Vásquez et al., 2019; Martínez Camargo, Morillo Coronado, Cepeda Mora, Mesa Fernández, 2020) y/o el lavado con agua corriente que remueva las sustancias inhibidoras solubles (Young y Young, 1999). En especies adaptadas a ambientes con incendios recurrentes se ha demostrado que los productos químicos resultantes de la combustión parcial o total de restos vegetales pueden actuar como precursores de la germinación (Keeley, 1987; Ghebrehiwot, Kulkarni, Light, Kirkman, van Staden, 2011). De esta forma las especies aseguran su reclutamiento luego de un incendio, natural o antrópico. El tratamiento artificial de semillas con humo proveniente de la carbonización de biomasa vegetal ha demostrado estimular la germinación en más de 1200 especies vegetales de 80 géneros diferentes (Brown y Botha, 2004), aunque no se ha probado en Anacardiáceas.

Por otra parte, hay especies que no producen semillas viables o éstas presentan condiciones de latencia muy complejas, por lo que la reproducción asexual o agámica es indispensable. Según Ingram (1993), el método de reproducción vegetativa más utilizado en la industria de propagación comercial de especies vegetales es mediante estacas o esquejes. Relacionado con esto, se encontró una importante cantidad de rebrotes producidos por Prosopis affinis (ñandubay), P. alba var. alba, P. nigra var. nigra y $P$. nigra var. ragosei (algarrobos), lo que indica que ésta puede ser una muy buena técnica para obtener material rejuvenecido para ensayos de enraizamiento (Lallana, Elizalde, Billard, Sabattini, Lallana, 1999). Considerando especies de la Familia Anacardiaceae, se citó a Schinus terebinthifolius como un ejemplar de gran potencial para ser aprovechado como estaca viva (Cardoso, Schwonke, Nachtigal, 2009). Los estudios realizados acerca del uso de promotores de enraizamiento han demostrado resultados contradictorios en especies arbustivas, dependiendo además del estado fenológico del ejemplar al momento de la recolección del material (Rodríguez Ferradá y Lemes Hernández, 2000; Latsague Vidala, Sáez Delgado, Hauenstein Barra, 2008). 
La elección de la forma más adecuada para la reproducción de una especie depende del objetivo deseado. En caso de proyectos de reforestación de ambientes naturales, la obtención de ejemplares con variabilidad genética es deseable, recomendándose la propagación por semillas. Sin embargo, para producción de plantas con fines ornamentales, la homogeneidad entre los ejemplares ofrece una ventaja en su manejo, sumado al menor tiempo requerido para la obtención de plantas adultas. Otras especies del género han demostrado resultados satisfactorios en los intentos de reproducción ex situ, como por ejemplo, S. areira, S. longifolius y S. molle (Codina et al., 2003).

Si bien existe información sobre la fenología y morfología de $S$. jonhstonii (Giorgetti et al., 2000; Steibel y Troiani, 2008), no existe literatura sobre la posibilidad de reproducción sexual y asexual de esta especie, información de interés para su incorporación en proyectos paisajísticos y de restauración de áreas degradadas. Los objetivos de este trabajo fueron: (1) establecer la existencia y el tipo de dormición en semillas; (2) determinar la viabilidad de las mismas; y (3) analizar la supervivencia de estacas en condiciones controladas y bajo distintos tratamientos. Los resultados obtenidos contribuyen a incrementar el conocimiento sobre la biología de esta especie, a fin de promover su conservación en los pastizales naturales y su reintroducción en áreas degradadas del Monte.

\section{MATERIALES Y MÉTODOS}

Recolección de material.- El material vegetal se recolectó en la Chacra Experimental Patagones, al sudoeste de la provincia de Buenos Aires (40 $39^{\prime}$ S, 62 ${ }^{\circ} 53^{\prime} \mathrm{O}$ ), dentro de la provincia Fitogeográfica del Monte (Cabrera, 1976). El clima es templado, semiárido, con lluvias concentradas en verano y otoño (promedio de precipitaciones total anual: $350,8 \mathrm{~mm}$ ).

Para el estudio se seleccionaron ejemplares adultos, de 1,5-2 $\mathrm{m}$ de altura, de poblaciones naturales y se recolectaron frutos maduros en los meses de noviembre y diciembre. Adicionalmente, en primavera, se recolectó material joven para la producción de estacas, cuando las plantas se encontraban en estado vegetativo (en activo crecimiento y sin yemas florales) y reproductivo (en floración plena).

Tratamientos pre-germinativos y germinación. - Los frutos recolectados se observaron bajo microscopio estereoscópico, a fin de seleccionar aquellos que no presentaran daño por insectos (Villagra, 2000). Posteriormente se lavaron en agua con lavandina (proporción 4:1) (Corkidi et al., 1991) y se sometieron a seis tratamientos: (1) semillas con exocarpo intacto (control; CE), (2) semillas sin exocarpo (desnudas; SE), (3) semillas desnudas con escarificación mecánica ( $\mathrm{SE}+\mathrm{EM}),(4)$ semillas desnudas con escarificación química $(\mathrm{SE}+\mathrm{EQ}),(5)$ semillas desnudas en remojo $(\mathrm{SE}+\mathrm{R})$ y (6) semillas desnudas con exposición a humo (SE + H) (Keeley, 1987; Casal, Prado, Reyes, Rivas, 2001; Torres, Long, Zalba, 2008). Para los tratamientos que incluyeron semillas desnudas, el exocarpo se eliminó de forma manual. La escarificación mecánica se realizó manualmente con papel de lija $\mathrm{N}^{\circ} 220$, mientras que la escarificación química se llevó a cabo mediante la inmersión de las semillas en ácido sulfúrico al 
10\%, durante 5 minutos (Pereira et. al, 2016). Las semillas remojadas permanecieron en agua a temperatura ambiente $\left(24^{\circ} \mathrm{C}\right)$ durante 4 días. El tratamiento con humo se realizó con un ahumador, con material vegetal seco encendido en su interior, y una bolsa plástica como receptáculo del mismo. Las semillas se introdujeron en la bolsa y se mantuvieron por 5 minutos bajo condiciones de saturación (Casal et al., 2001).

Las semillas tratadas se colocaron en 48 cajas de Petri ( 20 semillas/caja; $n=8$; $\mathrm{N}=960$ ) preparadas previamente con una capa de algodón sobre el cual se colocó un disco de papel de filtro. Las semillas se humedecieron con agua destilada y se colocaron en cámara de germinación, bajo condiciones de luz y temperatura (13 horas de luz a $25^{\circ} \mathrm{C}$ y 11 horas de oscuridad a $15^{\circ} \mathrm{C}$ ) similares a las que se presentan en septiembre y octubre, momento del año en que se produce la germinación de las semillas (Giorgetti et al., 2000). Diariamente se registró el número de semillas germinadas por réplica y tratamiento, tomando como criterio de germinación el momento en el cual se observó la emergencia de una radícula de $2 \mathrm{~mm}$ (Young y Young, 1999). Las semillas permanecieron en la cámara hasta que ya no se observó germinación.

Con los datos de semillas germinadas se calculó el Coeficiente de Velocidad de Germinación (CVG) según la siguiente fórmula de Kader (2005):

$$
\text { CVG }=\frac{\text { Número total de semillas germinadas }}{A_{1} T_{1}+A_{2} T_{2}+\ldots+A_{x} T_{x}}
$$

Donde $A$ es el número de semillas germinadas, $T$ el tiempo en días correspondiente a $A$, y los coeficientes $1,2, \ldots x$ corresponden al número de días desde el inicio hasta la evaluación final.

Viabilidad de semillas. - Una vez concluido el estudio de germinación, se analizó la viabilidad de las semillas no germinadas empleando cloruro de trifenil tetrazolio (TTC) al 0,5\%. Para esto se remojaron las semillas a fin de ablandar su cubierta, se cortaron por la mitad y se colocaron en frascos recubiertos con papel aluminio (debido a la fotosensibilidad del TTC). Se agregó la solución y se colocaron en estufa a $30^{\circ} \mathrm{C}$ durante 12 horas. Luego se observó con microscopio estereoscópico (Nikon, 10X) el grado y sitio de tinción de cada semilla (Peretti, 1994). Adicionalmente se registraron las semillas vacías.

Reproducción vegetativa.- Se obtuvieron estacas semileñosas de entre 15 y 20 $\mathrm{cm}$ de longitud, realizando un corte limpio, en bisel, con tijera de podar, justo por debajo de un nudo (Torres et al., 2008). Se emplearon 4 tratamientos $(\mathrm{n}=10)$ : estacas en estado vegetativo (1) con hormona y (2) sin hormona de enraizamiento; estacas en estado reproductivo (3) con hormona y (4) sin hormona. En cada caso, las estacas se colocaron en macetas en invernáculo con riego diario, respetando su polaridad y se registró su supervivencia durante 2 meses. La hormona empleada como enraizante fue el ácido alfa-naftalén acético (ANA) al 0,3\%. Las estacas se colocaron en un recipiente que contenía la hormona en polvo, cubriendo un centímetro de la misma, desde el área de corte (Torres et al., 2008). 
Análisis estadísticos. - Para cumplir con los supuestos de normalidad y homocedasticidad se transformaron los datos de porcentaje de germinación con $\sqrt{ }$ arco-seno (x) y los de reproducción vegetativa y el CVG con $\sqrt{ }(x+0,5)$ (Sokal y Rohlf, 1984). El diseño en todos los casos fue completamente aleatorizado. Los datos obtenidos en los estudios de germinación y viabilidad se analizaron mediante ANOVA simple, mientras que los derivados del estudio de reproducción vegetativa se analizaron con ANOVA doble. Para la comparación de medias se utilizó el test de Tukey, con un nivel de significación del 5\%. Todos los análisis se realizaron utilizando el paquete estadístico INFOSTAT (Di Rienzo et al., 2009).

\section{RESULTADOS}

Tratamientos pre-germinativos y germinación.- Este estudio se dio por finalizado tras 15 días en los cuales no se registró germinación, totalizando 100 días desde el inicio. Solo se observó germinación en semillas desnudas. En aquellas en las que se mantuvo el exocarpo intacto no se hallaron signos de emergencia de radícula durante el periodo de tiempo que duró el estudio. La Figura 1 muestra la secuencia de desarrollo desde la emergencia de la radícula hasta la aparición de las primeras hojas, en una semilla sin exocarpo y sin tratamiento.

No se encontraron diferencias significativas $(p>0,05)$ en los porcentajes de germinación entre los tratamientos que implicaron la remoción del exocarpo de las semillas. Solo se hallaron diferencias significativas $(\mathrm{p}<0,05)$ entre el tratamiento de semillas con exocarpo respecto de las semillas expuestas a los tratamientos sin exocarpo y sin exocarpo con escarificación mecánica (Fig. 2).

Las semillas tratadas con escarificación mecánica, remojo y humo fueron las primeras en germinar, dentro de los primeros 10 días de estudio, lo que se vio reflejado en su mayor valor de CVG en relación a los tratamientos con exocarpo y con escarificación química (Tabla 1). Las semillas que fueron expuestas a escarificación

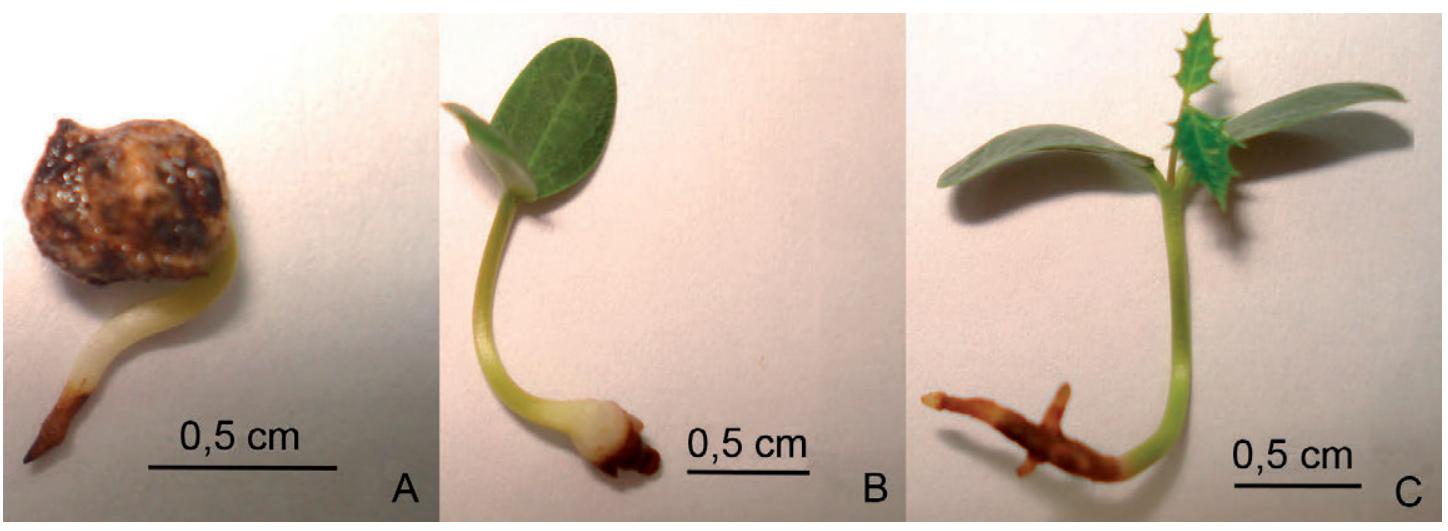

Fig 1. Germinación de Schinus johnstonii. A) Emergencia de radícula en semilla desnuda (sin exocarpo). B) Emergencia de cotiledones. C) Cotiledones y primeras hojas.

Fig. 1. Germination of Schinus johnstonii. A) Emergence of radicle in bare seed (without exocarp). B) Cotyledons emergence. C) Cotyledons and first leaves. 


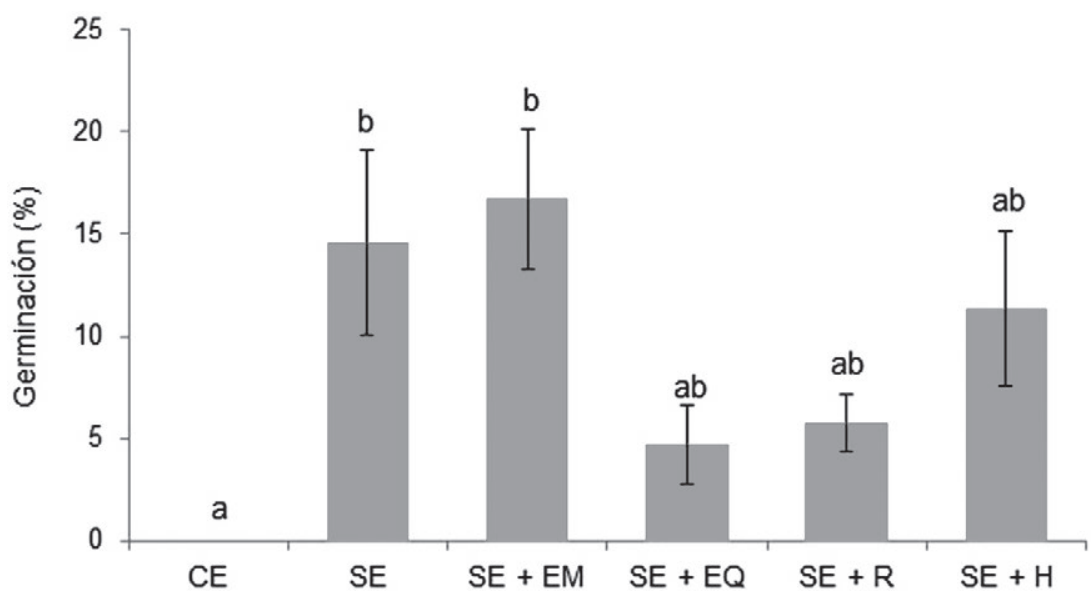

Fig. 2. Porcentaje de germinación de semillas de Schinus johnstonii bajo distintos tratamientos (promedio \pm error estándar; $n=8$ ). Letras distintas sobre los histogramas indican diferencias significativas entre tratamientos $(p<0,05)$. CE: con exocarpo; SE: sin exocarpo; SE + EM: sin exocarpo y con escarificación mecánica; SE + EQ: sin exocarpo y con escarificación química; SE + R: sin exocarpo y con remojo; SE + H: sin exocarpo y con humo.

Fig. 2. Germination percentage of Schinus johnstonii seeds under different treatments (average \pm standard error; $n=8)$. Different letters above the histograms indicate significant differences between treatments $(p<0.05)$. CE: with exocarp; SE: without exocarp; SE + EM: without exocarp and with mechanical scarification; SE + EQ: without exocarp and with chemical scarification; SE + R: without exocarp and with soaking; SE + H: without exocarp and with smoke.

mecánica germinaron en primer lugar, seguidas por las tratadas con humo y luego por las que fueron previamente remojadas. A los 10 días comenzaron a germinar las semillas sin exocarpo y a los 30 días las semillas del tratamiento químico (Fig. 3). Luego del día 85 ya no se registró germinación en ningún tratamiento.

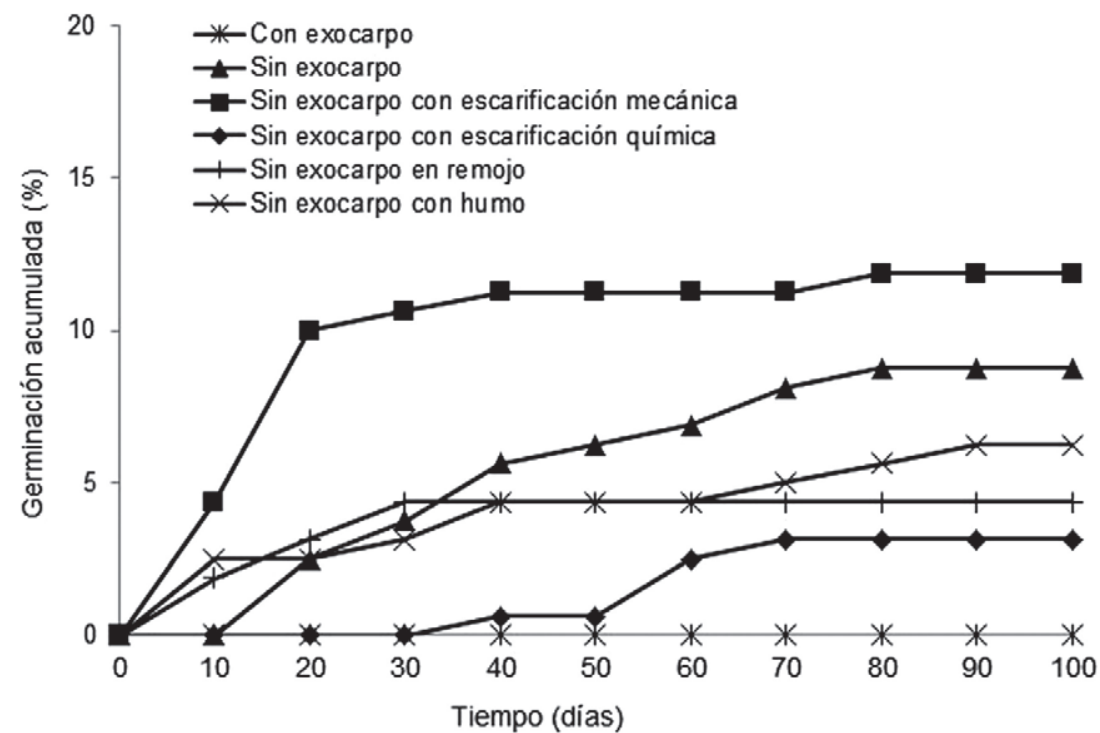

Fig. 3. Porcentaje de germinación acumulada en función del tiempo, para semillas de Schinus johnstonii bajo distintos tratamientos $(n=8)$.

Fig. 3. Cumulative germination percentage over time of Schinus johnstonii seeds under different treatments $(n=8)$. 
Tabla 1. Coeficiente de velocidad de germinación (CVG) para semillas de Schinus johnstonii bajo diferentes tratamientos pre-germinativos (promedio \pm error estándar; $n=8$ ). Letras distintas entre filas indican diferencias significativas entre tratamientos $(p<0,05)$.

Table 1. Coefficient of velocity of germination (CVG) for Schinus johnstonii seeds under different pre-germination treatments (average \pm standard error; $n=8$ ). Different letters between rows indicate significant differences between treatments $(p<0.05)$.

\begin{tabular}{ll}
\hline Tratamiento & CVG \\
\hline Con exocarpo & $0,00 \pm 0,00 \mathrm{a}$ \\
\hline Sin exocarpo & $0,05 \pm 0,01 \mathrm{ab}$ \\
\hline Sin exocarpo + escarificación mecánica & $0,08 \pm 0,01 \mathrm{~b}$ \\
\hline Sin exocarpo + escarificación química & $0,01 \pm 0,01 \mathrm{a}$ \\
\hline Sin exocarpo + remojo & $0,09 \pm 0,02 \mathrm{~b}$ \\
\hline Sin exocarpo + humo & $0,08 \pm 0,02 \mathrm{~b}$ \\
\hline
\end{tabular}

Viabilidad de semillas. - En general se observó que todos los tratamientos presentaron una proporción similar de semillas viables, no viables y vacías (Fig. 4; Tabla 2). Los menores porcentajes de semillas viables se registraron en aquellas asignadas al tratamiento con remojo, seguidas por las sometidas a escarificación. No hubo diferencias en las semillas no viables registradas en cada tratamiento, pero sí en el porcentaje de semillas vacías, encontrándose el mayor valor en las expuestas a humo y el menor en las que fueron remojadas. En función del número de semillas vacías

Tabla 2. Porcentaje de semillas de Schinus johnstonii viables, no viables y vacías, bajo diferentes tratamientos pre-germinativos (promedio \pm error estándar; $\mathrm{n}=8$ ). Letras distintas entre filas indican diferencias significativas entre tratamientos $(p<0,05)$.

Table 2. Percentage viable, non viable and empty seeds of Schinus johnstonii, under different pregermination treatments (average \pm standard error; $n=8$ ). Different letters between rows indicate significant differences between treatments $(p<0.05)$.

\begin{tabular}{|c|c|c|c|}
\hline Tratamiento & Viables (\%) & No viables (\%) & Vacías (\%) \\
\hline Con exocarpo & $23,1 \pm 3,1$ a & $37,5 \pm 3,3$ & $39,4 \pm 2,6 \mathrm{bcd}$ \\
\hline Sin exocarpo & $26,9 \pm 2,9 a b$ & $32,5 \pm 2,8 \quad a$ & $40,6 \pm 3,3 \mathrm{~cd}$ \\
\hline Sin exocarpo + escarificación mecánica & $41,4 \pm 4,9$ abc & $31,4 \pm 33,6 a$ & $27,1 \pm 4,8 a b$ \\
\hline Sin exocarpo + escarificación química & $36,4 \pm 6,4 b c$ & $33,6 \pm 6,2 \quad a$ & $30,0 \pm 4,3 a b c$ \\
\hline Sin exocarpo + remojo & $43,1 \pm 2,1 \mathrm{c}$ & $32,5 \pm 2,7 \quad a$ & $24,4 \pm 2,7 \quad a$ \\
\hline Sin exocarpo + humo & $21,2 \pm 2,7 a$ & $33,6 \pm 3,1 \quad a$ & $45,0 \pm 3,8 d$ \\
\hline
\end{tabular}

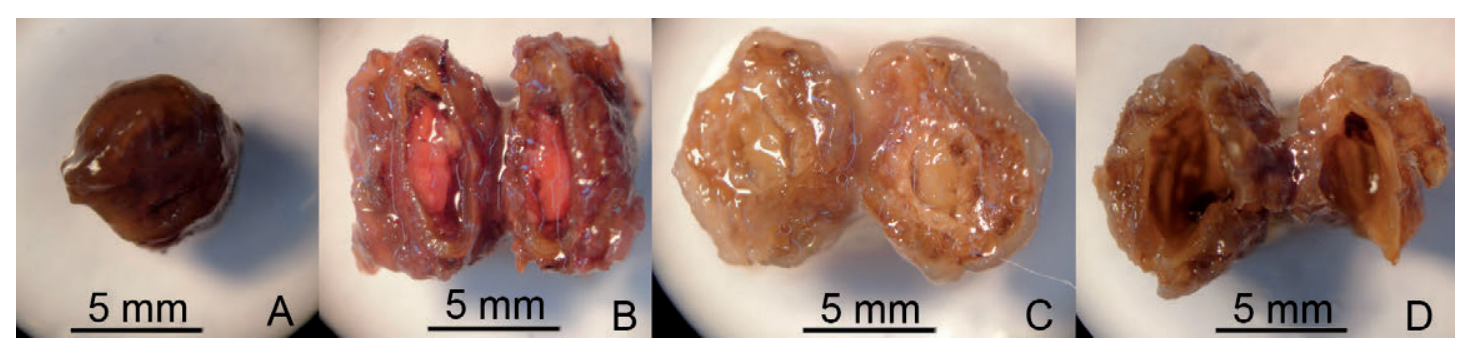

Fig. 4. Semillas desnudas (sin exocarpo) de Schinus johnstonii vistas al microscopio estereoscópico. A) Semilla entera. B) Semilla viable. C) Semilla no viable. D) Semilla vacía.

Fig. 4. Bare seeds (without exocarp) of Schinus johnstonii seen under a stereoscopic microscope. A) Whole seed. B) Viable seed. C) Non-viable seed. D) Empty seed. 
en cada tratamiento se recalcularon los porcentajes de germinación, alcanzándose los siguientes valores: $\mathrm{SE}+\mathrm{EM}=38 \%, \mathrm{SE}=32 \%, \mathrm{SE}+\mathrm{H}=25 \%, \mathrm{SE}+\mathrm{R}=21 \% \mathrm{y}$ $\mathrm{SE}+\mathrm{EQ}=15 \%$, sin variación en los resultados estadísticos.

Reproducción vegetativa.- Se observó rebrote de las estacas en todos los tratamientos con excepción de las producidas en estado reproductivo y con aplicación de hormona; sin embargo, no se encontraron diferencias significativas $(p>0,05)$ entre los cuatro tratamientos. Los porcentajes de supervivencia fueron $10 \pm 10 \%$ y $30 \pm$ $15 \%$, para las estacas en estado vegetativo con y sin hormona, respectivamente; y 20 $\pm 13 \%$ para el tratamiento restante.

\section{DISCUSIÓN}

Las semillas representan el único medio de dispersión y acceso a nuevos hábitats para muchas especies, y por ello se las considera como una parte integral y crucial de los ecosistemas (Pirk, 2007). La capacidad de germinar y establecerse, aún bajo condiciones de déficit hídrico, está asociada a la capacidad de colonización de ambientes áridos (Villagra et al., 2011). A su vez, la presencia de latencia en las semillas es considerada como una característica adaptativa necesaria para la supervivencia de las especies vegetales en estos ambientes (Villagra, 2004). Sin embargo, para fines de reproducción ex situ esta característica resulta un impedimento a resolver.

Schinus johnstonii es una especie adaptada a zonas áridas y semiáridas, por lo que, al igual que otras especies acompañantes, presenta dormición física en sus semillas. En este estudio se observó que este impedimento se pudo superar removiendo manualmente el exocarpo que las protege. Este mismo resultado se observó en $S$. molle y en $S$. areira, donde el porcentaje de germinación de semillas con exocarpo fue $0 \%$ y $1 \%$, respectivamente (Pereira et al., 2016; Zapater et al., 2018). En un estudio posterior donde se incubaron semillas de $S$. johnstonii sin ningún tratamiento pre-germinativo se obtuvo un porcentaje de germinación prácticamente nulo (0,3\%; Sosa, 2019).

En el presente estudio, sólo se hallaron diferencias significativas entre semillas con exocarpo y semillas sin exocarpo y sin exocarpo con escarificación mecánica, aunque estos últimos dos tratamientos no difirieron significativamente entre sí. Estos resultados son similares a los informados por Araoz y del Longo (2006) en Ziziphus mistol, otra especie leñosa de zonas áridas, quienes reportaron que la óptima germinación se obtuvo cuando la cubierta de la semilla fue removida completamente. En condiciones naturales, el efecto producido por las ráfagas de viento, que arrastran las semillas y las golpean contra las partículas del sustrato, sumado a la alternancia de temperaturas típica de ambientes semiáridos y la acción de microorganismos del suelo, promueven el deterioro de la testa, ejerciendo una escarificación natural, que deja zonas expuestas y aptas para la imbibición con agua y el intercambio gaseoso (Jara, Arangio, Moreno, Carmona, 2006). La falta de diferencia entre los demás tratamientos con semillas desnudas difiere de resultados informados en otras especies de Anacardiáceas. Por ejemplo, para $S$. molle la remoción del exocarpo junto a la escarificación ácida (Corkidi et al., 1991; Pereira et al., 2016) aumentaron la capacidad 
germinativa de las semillas, mientras que para $S$. areira, el remojo de las semillas desnudas fue el tratamiento con mejor resultado (Zapater et al., 2018).

En especies vegetales adaptadas al fuego, las sustancias químicas presentes en el humo pueden estimular la germinación, constituyendo una importante estrategia de supervivencia en ambientes sometidos a este disturbio (Keeley, 1987). Particularmente, el butenólido 3-metil-2H-furo [2,3-c] piran-2-ona ha sido identificado como el principal precursor de la germinación (Ghebrehiwot et al., 2011). En estudios realizados sobre los efectos del fuego en leguminosas arbustivas, se observó que el humo no estimuló la germinación de Ulex galli y Adenocarpus lainzzi, pero sí la aceleró en $U$. europaeus (Casal et al., 2001). Este resultado coincide con los hallados en este trabajo, en donde las semillas de $S$. johnstonii desnudas y tratadas con humo germinaron con mayor rapidez respecto al control y al tratamiento con ácido. Sin embargo, la exposición al humo no tuvo incidencia significativa sobre el porcentaje final de semillas germinadas. Esto contradice los trabajos realizados en la zona semiárida de Chile, donde se encontró que el humo estimuló la germinación de tres especies (Acacia caven, Baccharis vernalis y Trevoa quinquenervia) pero inhibió la de otras ocho, varias de las cuales son nativas dominantes del matorral (Lithrea caustica, Quillaja saponaria y Cryptocarya alba). Además, las semillas de la Anacardiácea $L$. caustica fueron particularmente inhibidas por el fuego (Figueroa y Jaksic, 2004). En cambio, para la especie leñosa Fabiana imbricata, a pesar de encontrarse un efecto estimulador del humo sobre la germinación, especialmente en semillas ya escarificadas, la combinación de este factor más la exposición al calor y la escarificación mecánica produjo los mejores resultados (Dudinszky y Ghermandi, 2013). Para conocer el efecto real del fuego sobre $S$. johnstonii, sería importante evaluar el conjunto de factores que afectan a las semillas expuestas a un incendio.

Los porcentajes de germinación alcanzados son relativamente bajos; sin embargo, son similares a los encontrados para otras especies leñosas de zonas semiáridas. Se ha reportado que la escarificación mecánica produjo un $7 \%$ de germinación en $P$. flexuosa (Villagra, 2000) y entre 4 y 5 \% en P. caldenia (Lerner y Peineti, 1996). Eliminando de los valores finales a las semillas que se encontraban vacías, los porcentajes de germinación alcanzados (15-38\%) son ligeramente superiores a los informados para S. molle (10\%; Pereira et al., 2016) y S. areira (35\%; Zapater, 2018).

El tiempo requerido para el inicio de la germinación en los distintos tratamientos (con excepción de las semillas con exocarpo y con tratamiento químico) coincide con lo informado para otras especies de la Familia. En $S$. molle la germinación se inició en los primeros 5-10 días (Corkidi et al., 1991), mientras que en Pistacia lenticus, a los 12 días (Verdú y García-Fayos, 1998). El tratamiento con ácido presentó un retraso considerable, lo que se condice con su menor $\mathrm{CVG}$, aunque la concentración empleada y el tiempo de exposición fueron similares a los recomendados para $S$. molle (Pereira et. al, 2016).

A pesar de encontrarse diferencias en los valores de semillas viables en cada tratamiento, esto no se relacionó con los porcentajes finales de germinación. El porcentaje de semillas viables con exocarpo no difirió del de aquellas desnudas, aunque sí lo hizo su germinación. De igual forma, las semillas sometidas al tratamiento de remojo mostraron un mayor porcentaje de viabilidad, pero esto no se vio reflejado 
en un incremento en la germinación. Estos resultados se encuentran comprendidos dentro del porcentaje de viabilidad de semillas informado para otra Anacardiácea, $P$. lentiscus (entre 20 y 45\%), para la cual el número final de semillas viables por planta fue función de la disponibilidad hídrica al momento de la formación de las semillas (Jordano, 1988). La fracción de la cosecha anual de frutos que llega a madurar es muy variable y depende de cuál haya sido la tasa de desarrollo de embriones en las semillas. Aun así, muchos frutos pueden alcanzar una madurez aparente conteniendo semillas no viables. La menor germinación en algunas especies, se puede atribuir fundamentalmente a la gran cantidad de semillas y frutos vanos, o a la inmadurez por un llenado deficiente (Jordano, 1988). En condiciones naturales, las plantas deben sincronizar sus ciclos de crecimiento y reproducción con un adecuado abastecimiento hídrico. Esto es especialmente importante en ambientes semiáridos, donde los eventos de lluvia son esporádicos o inexistentes, alternándose, de manera no previsible, años húmedos y años secos (Stritzler et al., 2007). La disponibilidad de agua durante el crecimiento de una planta madre afecta el desarrollo de sus semillas, alterando su capacidad germinativa positiva o negativamente (Maldonado, Pujado, Squeo, 2002). Esta información, junto a los resultados obtenidos en estudios con especies vegetales de zonas semiáridas o pertenecientes a la Familia Anacardiaceae, avalan los resultados de este estudio. De las semillas de $S$. johnstonii que no germinaron, al menos una cuarta parte estaban vacías y un tercio resultaron no viables. Esto podría deberse a un elevado déficit hídrico; ya que, en el año en que se formaron las semillas empleadas, se registró una precipitación anual extremadamente baja (195,5 $\mathrm{mm}$ ). En P. lenticus, el porcentaje de semillas viables se redujo a la mitad en un año con elevado estrés hídrico en comparación a un año con mayores precipitaciones (Jornado, 1988). La baja germinación de las semillas de Schinopsis balansae (27\%) se debió principalmente a la presencia de semillas vacías (Alzugaray, Carnevale, Salinas, Pioli, 2007). Estudios realizados en frutos maduros de Pistacia terebinthus y P. lenticus revelaron que el número de semillas vacías varió desde 5\% hasta $45 \%$ (García-Fayos et al., 2001), mientras que en S. areira este valor ascendió a 73\% (Zapater et al., 2018). Jordano (1988) también mencionó casos de frutos con semillas no viables en los géneros Rhus, Anacardium y Spondias. La inviabilidad de los frutos puede ocurrir por falta de polinización, desarrollándose frutos partenocárpicos sin semillas viables; por aborto del embrión en flores ya polinizadas; o por predación a partir de invertebrados (Jordano, 1988). En el presente estudio, se encontró además una gran cantidad de drupas comidas. A pesar que los frutos con daños visibles se descartaron, algunos pudieron pasar desapercibidos afectando la germinación final. La infestación por brúquidos (Coleoptera, Bruchidae), que dañan directa o indirectamente al embrión, es habitual en semillas de especies de zonas áridas, habiéndose encontrado en frutos y semillas de S. areira (Zapater et al., 2018) y en varias especies de Prosopis (Villagra et al., 2004; Mazzuferi y Conles, 2005).

Conservar las características intrínsecas de valor de un genotipo o la aptitud para prosperar en condiciones culturales particulares siguen siendo las razones fundamentales del uso de la propagación vegetativa en la multiplicación comercial de las plantas (Barbat, 2006). En S. terebinthifolius se halló que el porcentaje medio de supervivencia de estacas fue de solo $23 \%$, concluyéndose, de todos modos, que las 
estacas caulinares de esta especie son promisorias para la propagación vegetativa (Cardoso et al., 2009). Estos valores son similares a los obtenidos en el presente estudio. Los resultados relativos al momento de recolección de las estacas no son concluyentes, aunque se observó rebrote en estacas recolectadas cuando la planta se encontraba en estado reproductivo. Esto contradice a Barbat (2006), quien informó que, en general, la aptitud al enraizamiento es mayor durante la fase vegetativa, tendiendo a desaparecer durante la fase reproductiva. Los resultados obtenidos también se oponen a los datos experimentales informados para leñosas como Ruta graveolens (Rodríguez Ferradá y Lemes Hernández, 2000) y Tarasa reichei (Gómez, Hahn, San Martín, Vizcarra, 2010). Sin embargo, coinciden con los resultados observados en estacas semileñosas de Pavonia cymbalaria, donde se observó mayor enraizamiento en esquejes semileñosos recolectados durante la fase reproductiva (Torres et al., 2008). Al respecto, Di Benedetto (2004) recomienda la recolección del material en primavera para especies de difícil enraizamiento. El contenido endógeno de las hormonas, entre ellas las auxinas responsables de la inducción de las raíces adventicias, varía según la época del año, siendo mayor en primavera, luego del reposo invernal, cuando hay un activo crecimiento de los brotes, favoreciendo el enraizamiento de las estacas (Sisaro, 2016). A pesar que el material recolectado provenía de individuos en distinto estado fenológico, un contenido hormonal similar de las plantas al momento de la recolección (primavera) explicaría la falta de diferencias encontradas en los tratamientos.

Hay especies que dependen de los tratamientos con auxinas naturales o de síntesis para lograr el enraizamiento (Barbat, 2006). En este estudio, S. johnstonii no mostró diferencias entre los tratamientos con y sin hormona enraizante. Estos resultados coinciden con los obtenidos para estacas semileñosas de Prosopis alba, donde la aplicación y las dosis de hormona no causaron efecto significativo sobre la supervivencia, enraizamiento y crecimiento de las estacas (Oberschelp y Marcó, 2010). Resultados similares fueron citados para otras especies leñosas y semileñosas como Gliricidia sepium, Salix humboldtiana y P. cymbalaria (Giraldo, Ríos, Polanco, 2009; Torres et al, 2008). De todas formas, los porcentajes de enraizamiento fueron bajos, por lo que debería evaluarse la aplicación de la hormona en mayores concentraciones, o el empleo de otras hormonas, como el ácido indolbutírico (AIB) o el ácido-3-indol acético (AIA). En algunos casos la combinación de AIA junto a AIB incrementa los porcentajes de enraizamiento y supervivencia (Ramos Gavilanes, Cruz Rosero, Morante Carriel, Villacís, 2006).

Schinus johnstonii mostró, en condiciones de invernadero, un bajo porcentaje de rebrote de los esquejes colectados, convirtiendo a la propagación vegetativa en una estrategia poco efectiva con los tratamientos aplicados. Una buena selección del esqueje es esencial para el éxito reproductivo. La edad de la planta madre puede ser un factor determinante en la formación de raíces adventicias, ya que el material proveniente de plantas jóvenes, que aún presenten tejidos semileñosos de reciente formación, tiene mayor potencialidad rizogénica (Barbat, 2006). En caso de que las plantas ya sean adultos establecidos se recomienda elegir material que haya sido producido en la misma temporada para que posea características de juvenilidad y así facilitar su enraizamiento adventicio (Sisaro, 2016). Además, se debe considerar el estado sanitario y vigor de la planta madre. En este estudio se emplearon ejemplares 
adultos, aparentemente sanos, pero no se consideró su edad debido a la dificultad de estimarla visualmente en poblaciones naturales.

\section{CONCLUSIONES}

Las semillas de $S$. johnstonii presentaron dormición física impuesta por la cubierta. La remoción del exocarpo es suficiente para obtener los mayores porcentajes de germinación, en un tiempo reducido, sin necesidad de otro tratamiento adicional.

La viabilidad de las semillas fue más elevada en los tratamientos en los que se removió el exocarpo, con excepción de aquel que incluyó además la aplicación de humo. Se observó, en general, una importante proporción de semillas no viables o vacías.

La supervivencia de las estacas (reproducción vegetativa) no fue afectada por el uso de hormonas promotoras del enraizamiento ni por el estado fenológico de la planta madre (vegetativo o reproductivo). Debido al bajo éxito de implantación obtenido se recomienda profundizar en el conocimiento de esta técnica, realizando nuevos estudios que contemplen otras hormonas y la edad de la planta madre al momento de la recolección del material.

\section{AGRADECIMIENTOS}

Las autoras agradecen al personal de la Chacra Experimental Patagones (Ministerio de Agroindustria de la Provincia de Buenos Aires) por la ayuda brindada en las tareas de recolección del material vegetal.

\section{BIBLIOGRAFÍA}

Alzugaray, C., Carnevale, N., Salinas, A. y Pioli, R. (2007). Factores bióticos y abióticos que afectan la calidad de las semillas de Schinopsis balansae Engl. y Aspidosperma quebracho-blanco Schltdl. Revista Iberoamericana de Micología 24: 142-147. https://doi.org/10.1016/S1130-1406(07)70030-X

Araoz, S. D. y Del Longo, O. T. (2006). Tratamientos pregerminativos para romper la dormición física impuesta por el endocarpo en Ziziphus mistol Grisebach. Revista de Ciencias Forestales, Quebracho 13: 56-65.

Barbat, T. (2006). La multiplicación de las plantas. Viveros II. Extras 1: 33-43.

Bogino, S., Gómez, M., Ávila, A., Furlán, Z., Escudero, S., Corral, A. y Molinero, H. (2002). La forestación con especies nativas: análisis de un caso promisorio en la provincia de San Luis. XVII Jornadas Forestales de Entre Ríos, Concordia. Entre Ríos, Argentina.

Brown, N. A. C. y Botha, P. A. (2004). Smoke seed germination studies and a guide to seed propagation of plants from the major families of the Cape Floristic Region, South Africa. South African Fournal of Botany 70 (4):559-581. https://doi. org/10.1016/S0254-6299(15)30194-0 
Cabrera, A. L. 1976. Regiones fitogeográficas Argentinas. En: Ferreira Sobral, E.F. (Ed.). Enciclopedia Argentina de Agricultura y Fardinería (pp. 1-85). Buenos Aires: ACME.

Cardoso, J. H, Schwonke, A. B. y Nachtigal, J. C. (2009). A práctica agroforestal de estacas vivas com Aroeira vermelha (Schinus terebinthifolius RADDI): resultados preliminares sobre os efeitos dos tratamentosdiämetro e incisao na casca. Universidade Federal de Pelotas e estagiário na Embrapa Clima Temperado, Endereco. Brasilia, Brasil, 4 pp.

Casal, M., Prado, S., Reyes, O. y Rivas, M. (2001). Efecto del fuego sobre la germinación de varias especies leguminosas arbustivas. III Congreso Forestal Español, Sociedad Española de Ciencias Forestales, Junta de Andalucía, pp. 475-481.

Codina, R. A., Carrieri, S. A., Manzano, E. R. y Fioretti, S. B. (2003). Paisajismo sustentable en zonas áridas especies útiles para parquizaciones xéricas. Revista de la Facultad de Ciencias Agrarias de la Universidad de Cuyo 35 (2): 33-44.

Corkidi, L., Cacho, S. y Búrquez, A. (1991). Dispersión del Pirú (Schinus molle L., Anacardiaceae) por aves en Teotihuacán, México. Acta Botánica Mexicana 15: 17-22. DOI:10.21829/abm15.1991.617

Di Benedetto, A. H. (2004). Cultivo intensivo de plantas ornamentales: Bases científicas y tecnológicas, $1^{\circ}$ Edición. Editorial de la Facultad de Agronomía, Buenos Aires.

Di Rienzo, J. A., Casanoves, F., Balzarini, M. G., González, L., Tablada, M. y Robledo, C. W. INFOSTAT versión 2009. Grupo INFOSTAT, FCA, Universidad Nacional de Córdoba, Argentina.

Doria, J. (2010). Revisión bibliográfica. Generalidades sobre las semillas: su producción, conservación y almacenamiento. Cultivos Tropicales 31 (1): 74-85.

Dudinszky, N. y Ghermandi, L. (2013). Fire as a stimulant of shrub recruitment in northwestern Patagonian (Argentina) grasslands. Ecological Research 28: 981-990. DOI: $10.1007 / \mathrm{s} 11284-013-1080-7$

Figueroa, J. A. y Jaksic, F. M. (2004). Latencia y banco de semillas en plantas de la región mediterránea de Chile central. Revista Chilena Historia Natural 77 (1): 201-215. http://dx.doi.org/104067/S0716-078X2004000100016

García-Fayos, P., Gulias, J., Martínez, J., Marzo, A., Melero, J. P., Traveset, A., Veintimilla, P., Verdú, M., Cerdá, V., Gasque, M. y Medrano, H. (2001). Bases ecológicas para la recolección, almacenamiento y germinación de semillas de especies de uso forestal de la Comunidad Valenciana. Valencia: Banc de Llavors Forestals.

Ghebrehiwot, H. M., Kulkarni, M. G., Light, M. E., Kirkman, K. P. y van Staden, J. (2011). Germination activity of smoke residues in soils following a fire. South African Fournal of Botany 77: 718-724. DOI: 10.1016/j.sajb.2011.03.006

Giorgetti, H. D., Manuel, Z., Montenegro, O. A., Rodríguez, G. D. y Busso, C. A. (2000). Phenology of some herbaceous especies in central semiarid Argentina. Phyton International Fournal of Experimental Botany 69: 91-108.

Giorgetti, H. D., Montenegro, O. A., Rodríguez, G. D. y Busso, C. A. (2006). Tasas de crecimiento relativo en gramíneas perennes de diferente palatabilidad en 
los pastizales del centro de Argentina. Revista Argentina de Producción Animal 26: 199-200.

Giraldo, L. A., Ríos, H. F. y Polanco, M. F. (2009). Efecto de dos enraizadores en tres especies forestales promisorias para la recuperación de suelos. Revista de Integración Agraria y Ambiental 1: 41-47. https://doi.org/10.22490/2145653.1966

Gómez, P., Hahn, S., San Martín, J. y Vizcarra, G. (2010). Propagación vegetativa de Tarasa reichei Phil. mediante enraizamiento de estacas. Chloris chilensis Año $13, \mathrm{~N}^{\mathrm{o}} 1$.

Ingram, D. (1993). Landscape Plant Propagation Workbook: Propagation by Cuttings. Florida Cooperative Extension Service, Institute of Food and Agricultural Sciences, USA: University of Florida.

Jara, P. A, Arangio, G., Moreno, R. y Carmona, M. R. (2006). Factores abióticos que influencian la germinación de seis especies herbáceas de la zona árida de Chile. Revista Chilena de Historia Natural 79: 309-319. https://dx.doi.org/10.4067/S0716078X2006000300003

Jordano, P. (1988). Polinización y variabilidad de la producción de semillas en Pistacia lentiscus L. (Anacardiaceae). Anales del Fardín Botánico de Madrid 45: 213-231.

Kader, M. A. (2005). A comparison of seed germination calculation formulae and associated interpretation of resulting data. Fournal and proceedings of the Royal Society of New South Wales138: 65-75.

Keeley, J. E. 1987. Role of fire in seed germination of Woody taxa in California chaparral. Ecology 68: 434-443. DOI: 10.2307/1939275

Lallana, M. C., Elizalde, J. H. I., Billard, C., Sabattini, R. A. y Lallana, V. H. (1999). Capacidad de rebrote y tasa de crecimiento en individuos de Prosopis spp. en un Monte nativo del departamento La Paz, Entre Ríos. En: Actas IV Jornadas Técnicas Forestales del Parque Chaqueño y II Jornadas de la Asociación Argentina de Prosopis. Entre Ríos, Argentina, pp. 56-64.

Latsague Vidala, M., Sáez Delgado, P. y Hauenstein Barra, E. (2008). Inducción de enraizamiento en estacas de Berberidopsis corallina con ácido indolbutírico. Bosque 29 (3): 227-230. https://dx.doi.org/10.4067/S0717-92002008000300006

Li, X., Baskin, J. M. y Baskin, C. C. (1999a). Anatomy of two mechanisms of breaking physical dormancy by experimental treatments in seeds of two North American Rhus species (Anacardiaceae). American Fournal of Botany 86 (11): 1505-1511.

Li, X., Baskin, J. M. y Baskin, C. C. (1999b). Physiological dormancy and germination requirements of seeds of several North American Rhus species (Anacardiaceae). Seed Science Research 9: 237-245. https://doi.org/10.1017/S0960258599000240

León-Lobos, P. M. y Kalin- Arroyo, M. T. (1994).Germinación de semillas de $\mathrm{Li}$ threa caustica (Mol.) H. et A. (Anacardiaceae) dispersadas por Pseudalopex spp. (Canidae) en el bosque esclerófilo de Chile central. Revista Chilena de Historia Natural 67: 59-64.

Lerner, P. y Peinetti, R. (1996). Importance of predation and germination losses from the seed bank of caldén (Prosopis caldenia). Fournal of Range Management 49: 147-150. DOI: 10.2307/4002685

Maldonado, C., Pujado, E. y Squeo, F. A. (2002). El efecto de la disponibilidad de agua durante el crecimiento de Lycopersicon chilense sobre la capacidad de 
sus semillas para germinar a distintas temperaturas y concentraciones de manitol y NaCl. Revista Chilena de Historia Natural 75: 651-660. https://dx.doi. org/10.40677s0716-078X2002000400002

Martín, G. O., Nicosia, M. G., Colombo, M. y Lucas, J. (2001). Fenología de floración y fructificación en leñosas nativas del Chaco Semiárido de Tucumán y algunas consideraciones para su aprovechamiento forrajero. II Reunión de Producción Vegetal del NOA, Tucumán, Argentina, p. 325-334.

Martínez Camargo, M. A., Morillo Coronado, A. C., Cepeda Mora, E. y Mesa Fernández, S. Y. (2020). Evaluation of pregerminative treatments in gulupa seeds (P. edulis f. edulis Sims). Revista Brasileira de Fruticultura 42 (3): 1-9. https://doi. org/10.1590/0100-29452020590

Mazzuferi, V. y Conles, M. (2005). Insectos y hongos que afectan las semillas de Prosopis. En: Verzino, GE, Joseau, MJ. (Eds.). El Banco Nacional de Germoplasma de Prosopis (pp. 69-78), Córdoba: Facultad de Ciencias Agropecuarias, Universidad Nacional de Córdoba.

Oberschelp, G. P. y Marcó, M. A. (2010). Efecto del ácido 3-indolbutírico sobre el enraizamiento adventicio y la altura de plantines clonales de Prosopis alba Grisebach. Quebracho 18: 112-119.

Pece, M. G., Sobrero, M. T., Acosta, M. y Rossi, F. (2014). Tratamientos pregerminativos en Geoffroea decorticans (gillies ex hook. \& arn.) Burkartvar. Decorticans Foresta Veracruzana 16 (2): 31-36.

Peláez, D. V., Bóo, R. M. y Elía, O. R. (1996). The germination and seeedling survival of Condalia microphylla Cav. in Argentina. Fournal of Arid Enviroments 32: 173-179.

Pensiero, J., Muñoz, J. D. y Martínez, V. (2005). Alternativas de sustentabilidad del bosque nativo del espinal. Proyecto Bosques Nativos y Áreas Protegidas. Proyecto de Investigación Aplicada a los Recursos Forestales Nativos (PIARFON).

Pereira, M. P., Corrêa, F. F., Polo, M., de Castro, E. M., Cardoso, A. A. y Pereira, F. J. (2016). Seed germination of Schinus molle L. (Anacardiaceae) as related to its anatomy and dormancy alleviation. Seed Science Research 26 (4): 351-361. DOI: $10.1017 / \mathrm{S} 0960258516000167$

Peretti, A. (1994). Manual para el análisis de semillas. Buenos Aires: INTA, Hemisferio Sur.

Pirk, G. I. (2007). Granivoría por hormigas del género Pogonomyrmex en el Monte Central: Consumo de semillas e impacto sobre el banco del suelo. Tesis Doctoral. Facultad de Ciencias Exactas, Universidad de Buenos Aires. Buenos Aires, Argentina, $144 \mathrm{pp}$.

Ramos Gavilanes, L., Cruz Rosero, N., Morante Carriel, J. y Villacís, O. (2006). Empleo de hormonas (ANA y AIB) estimuladoras del enraizamiento para la propagación vegetativa de Chlorophora tinctoria (L) Gaud (moral fino) en el litoral ecuatoriano. Foresta Veracruzana 8 (1): 9-12.

Rasmussen, A. G. y Wright, H. A. (1988). Germination requirements of flameleaf sumac. Fournal of Range Management 41 (1): 48-52. 
Rodríguez Ferradá, C. A. y Lemes Hernández, C. M. (2000). Estudio de propagación vegetativa de la ruda (Ruta graveolens L.). Revista Cubana de Plantas Medicinales 5 (2): 9-56.

Silva-Luz, C. L., Pirani, R. J., Mitchell, D. J., Daly, D., do Valle Capelli, N., Demarco, D., Pell, S. K. y Plunkett, G. M. (2018). Phylogeny of Schinus L. (Anacardiaceae) with a new infrageneric classification and insights into evolution of spinescence and floral traits. Phylogenetics and Evolution 133: 302-351. https://doi.org/10.1016/ j.ympev.2018.10.013

Sisaro, D. (2016). Propapagación vegetativa por medio de estacas de tallo. Ediciones INTA, Hurlingham, Buenos Aires.

Sokal, R. R y Rohlf, F. J. (1984). Biometry. New York: Freeman, WH y Co.

Sosa, C. N. (2019). Evaluación del uso del cutting como sustrato en la propagación de cinco especies nativas de Monte para su aplicación en restauración ecológica. Tesis de grado, Universidad Nacional del Comahue, $60 \mathrm{pp}$.

Steibel, P. E. y Troiani, H. O. (2008). La identidad de Schinus fasciculatus var. arenicola y rehabilitación de $S$. sinuatus (Anacardiaceae). Boletín de la Sociedad Argentina de Botánica 43 (1-2): 157-166.

Stritzler, N. P., Petruzzi, H. J., Frasinelli, C. A., Veneciano, J. H, Ferri, C. M. y Viglizzo, E. F. (2007).Variabilidad climática en la Región Semiárida Central Argentina. Adaptación tecnológica en sistemas extensivos de producción animal. Revista Argentina de Producción Animal 27: 113-125.

Torres, Y. A., Long, M. A. y Zalba, S. M. (2008). Reproducción de Pavonia cymbalaria (Malvaceae), una especie nativa con potencial ornamental. Phyton, International Fournal of Experimental Botany 77: 151-161.

Vásquez, W., Pupiales, P., Viteri, P., Sotomayor, A., Feican, C., Campaña, D. y Viera, W. (2019). Escarificación química y aplicación de ácido giberélico para la germinación de semillas de cultivares de mora (Rubus glaucus Benth). Interciencia 44 (3): 161-166.

Vega Riveros, C., Meglioli, P. A. y Villagra, P. E. (2011). Prosopis alpataco Phil. (Fabaceae, Mimosoideae). Kurtziana 36 (2): 53-64.

Verdú, M. y García-Fayos, P. (1998). Ecological causes, function, and evolution of abortion and parthenocarpy in Pistacia lentiscus (Anacardiaceae). Canadian Fournal of Botany 76 (1): 134-141.

Villagra, P. E. (2000). Aspectos ecológicos de los algarrobales argentinos. Multequina 9 (2): $35-51$.

Villlagra, P. E, Cony, M. A., Mantován, N. G., Rossi, B. E., González Loyarte, M. M., Villalba, R. y Marone, L. (2004). Ecología y Manejo de los Algarrobales de la Provincia Fitogeográfica del Monte. En: Arturi, M.F., Frangi, J.L., Goya, J.F. (Eds). Ecología y Manejo de Bosques Nativos de Argentina (32 pp.). Buenos Aires: Editorial Universidad Nacional de La Plata.

Villagra P. E., Defosse, G. E., del Valle, H. F., Tabeni, S., Rostagno, M., Cesca, E. y Abraham, E. (2009). Land use and disturbance effects on the dynamics of natural ecosystems of the Monte Desert: Implications for their management. Fournal of Arid Environments 73: 202-211. DOI:10.1016/j.jaridenv.2008.08.002 
Villagra, P. E., Giordano, C., Álvarez, J. A., Cavagnaro, J. B., Guevara, A., Sartor, C., Passera, C. B. y Greco, S. (2011). Ser planta en el desierto: estrategias de uso de agua y resistencia al estrés hídrico en el Monte Central de Argentina. Ecología Austral 21 (1): 9-42.

Wannan, B. (2006). Analysis of Generic Relationships in Anacardiaceae. Blumea Fournal of Plant Taxonomy and Plant Geography 51 (1): 165-195. DOI:10.3767/ 000651906 X622427

Young, J. A. y Young, C. G. (1999). Seed of Wildland Plants.Collecting, processing and germinating.Portland, USA: Timber Press.

Zapater, M. A., Alemán, M. M., Lozano, E. C., Aquino, V. H., Flores, C. B., Gil, M. N., Villada, J. y Araya, G. (2018). Aspectos reproductivos de Schinus areira L. (Anacardiaceae) en Argentina. Boletín de la Sociedad Argentina de Botánica 53 (4): 619-631. DOI:10.31055/1851.2372.v53.n4.21984 